\title{
Millipedes (Diplopoda) from the Rostov-on-Don Region, southern Russia
}

\section{Авупарноногие многоножкки (Diplopoda) из Ростовской обцасти (юг России)}

\author{
A.P. Evsyukov ${ }^{1}$, S.I. Golovatch ${ }^{2}$ \\ А.П. Евсюков ${ }^{1}$ С.И. Головач ${ }^{2}$
}

${ }^{1}$ Лицей № 1 «Классический», ул. Балакирева 32, Ростов-на-Дону 344004 Россия.
${ }^{1}$ Lyceum №. 1 “Classical”, Balakireva Str. 32, Rostov-on-Don 344004 Russia.
${ }^{2}$ Институт проблем экологии и эволюции РАН, Ленинский просп. 33, Москва 119071 Россия.
${ }^{2}$ Institute for Problems of Ecology and Evolution, Russian Academy of Sciences, Leninsky prosp. 33, Moscow 119071 Russia.

KEY WORDS: Diplopoda, faunistics, Rostov-on-Don Region, southern Russia.

КЛЮЧЕВЫЕ СЛОВА: Diplopoda, фаунистика, Ростовская область, Южная Россия.

ABSTRACT. The millipede fauna of the Rostovon-Don Region is reviewed and shown to comprise at least 17 species, eleven of which are new to the regional list, including five new to the fauna of Russia: $L o$ phoproctus lucidus (Chalande, 1888), Strongylosoma jaqueti Verhoeff, 1898, Polydesmus stuxbergi Attems, 1907, Brachydesmus jubatus Attems, 1907 and Megaphyllum transsylvanicum (Verhoeff, 1897). Faunistic records of all of the species in the Region are presented and mapped.

РЕЗЮМЕ. Дан обзор фауны двупарноногих многоножек Ростовской области, которая включает по крайней мере 17 видов. Из них 11 впервые приведены для исследуемой территории, в т.ч. пять новые для фауны России: Lophoproctus lucidus (Chalande, 1888), Strongylosoma jaqueti Verhoeff, 1898, Polydesmus stuxbergi Attems, 1907, Brachydesmus jubatus Attems, 1907 и Megaphyllum transsylvanicum (Verhoeff, 1897). Представлены фаунистические находки и карты с указанием распространения всех этих видов в области.

\section{Introduction}

For a very long time, the steppe zone lying in the south of the Ukraine and Russia, including the Rostovon-Don Region, has been subjected to large-scale anthropogenic transformations such as agri- and horticulture, shelter belts, irrigation systems, infrastructure etc. [Gvozdetsky, 1968]. These transformations apply to all steppe ecosystems, definitely affecting Diplopoda as well.

Millipedes are one of the major components of soil fauna in temperate regions, but up to the present the millipede fauna of the Rostov-on-Don Region has been poorly investigated. Only very few papers contain information concerning the Region's diplopods [Lokshina, 1969; Prishutova, 1986]. Thus, Lokshina [1969] recorded the following species: Nopoiulus kochii (Gervais, 1847) (referred to as $N$. venustus), Unciger transsilvanicus (Verhoeff, 1899), Brachyiulus jawlowskii Lohmander, 1928, Megaphyllum rossicum (Timotheew, 1897) (referred to as Chromatoiulus rossicus) and Rossiulus kessleri (Lohmander, 1927) (referred to as Sarmatoiulus kessleri). Prishutova [1986] focused on the millipedes from the south of the Region: $U$. transsilvanicus, M. rossicum, R. kessleri, B. jawlowskii and four closer unidentified species: Julus sp., Cylindroiulus sp., Polydesmida gen. sp. and Blaniulidae gen. sp. More recently, Cylindroiulus cambio Korsós \& Read, 1994 has been described from the Botanical Garden in the city of Rostov-on-Don [Korsós \& Read, 1994], and further faunistic records of $N$. kochii have also been provided [Golovatch \& Enghoff, 1990; Golovatch \& Matyukhin, 2011].

\section{Material and methods}

Material for the present paper was collected in 20042011 in different places and habitats of the Rostov-onDon Region. Specimens were hand-collected or taken by pitfall trapping. The material has been shared between the collections of the Zoological Museum of the Moscow State University, Moscow (ZMUM), the Museum of the Zoological Department of the Southern Federal University, Rostov-on-Don (MZDR), and of the first author (AE). 


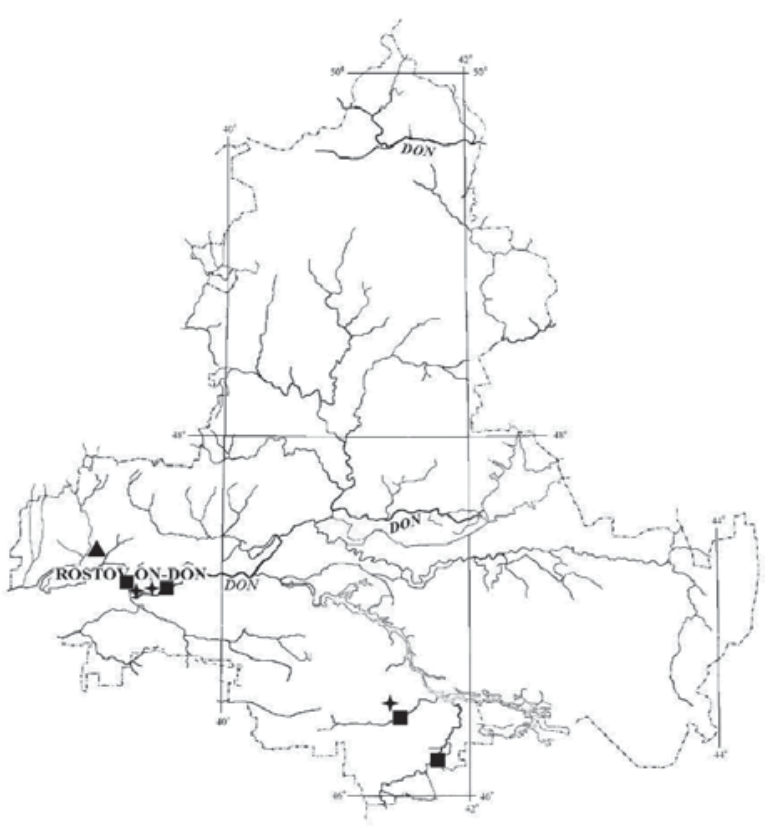

\section{Taxonomic part}

ORDER POLYXENIDA

\section{Family LOPHOPROCTIDAE}

Lophoproctus lucidus (Chalande, 1888) Map 1.

MATERIAL. 3 ex. (AE), Rostov-on-Don Region, Salsk Distr., near Salsk, woodland, under tree branches, 30.IV.2008; leg. A.P. Evsyukov \& D.D. Khisametdinova; 4 ex. (ZMUM), 1 ex. (MZDR), 1 ex. (AE), Myasnikovsk Distr., near Nedvigovka, Kamennaya

Map 1. Distribution of millipedes in the Rostov-on-Don Region: square - Strongylosoma kordylamythrum; triangle - S. jaqueti; cross - Lophoproctus lucidis.

Карта 1. Распространение двупарноногих многоножек на территории Ростовской области: квадрат — Strongylosoma kordylamythrum; треугольник - S. jaqueti; крест - Lophoproctus lucidis.
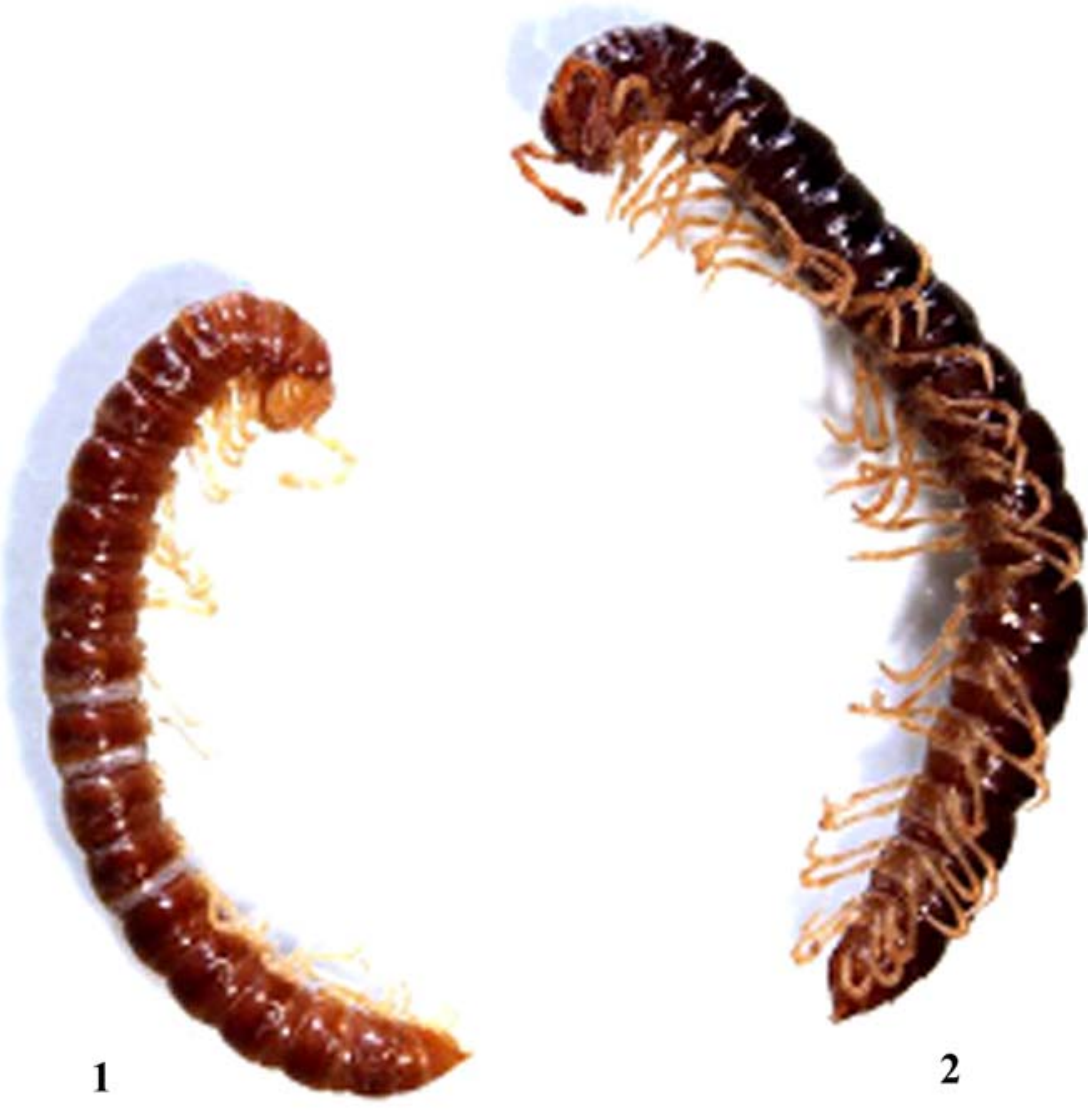

2

Figs 1 \& 2. Strongylosoma kordylamythrum, samples from the Rostov-on-Don Region (left) and Adygeya, NW-Caucasus (right), respectively. Photos by Alexey Tikhonov. Photographed not to scale.

Рис. 1 и 2. Strongylosoma kordylamythrum, экземпляры соответственно из Ростовской области (слева) и Адыгеи, Сев.-Зап. Кавказ (справа). Фото Алексея Тихонова. Без масштаба. 
gully, steppe, under stones, 12.IV.2009, A.P. Evsyukov, D.D Khisametdinova, V.Yu. Shmatko, D.D. Volkova \& I.L. Gorbenko; 3 ex. (AE), Neklinovsk Distr., near Merzhanovo, shelter belt, leaf litter, 6.VI.2009, leg. A.P. Evsyukov \& D.D. Khisametdinova; 11 ex. (AE), Neklinovsk Distr., near Morskoy Chulek, steppe, under stones, 18.V.2010, leg. A.P. Evsyukov, D.D. Khisametdinova \& V.Yu. Shmatko.

REMARKS. Above are the first records of this Mediterranean species in Russia. The geographically closest reports are from the southern Ukraine, including the Crimea [Chornyi \& Golovatch, 1993].

\section{ORDER POLYDESMIDA}

\section{Family PARADOXOSOMATIDAE}

Strongylosoma kordylamythrum Attems, 1898 Figs 1 \& 2; Map 1.

MATERIAL. $60^{7} \sigma^{7}, 7$ 우 (ZMUM), Rostov-on-Don Region, Salsk Distr., Salsk, 2.V.2004; 2 ㅇ (AE), same locality, 3.V.2004; $3 \mathrm{O}^{7} \mathrm{O}^{7}$ (AE), same locality, 16.VI.2008; $6 \mathrm{O}^{7} \mathrm{O}^{7}, 1$ \% (AE), same locality, 2.V.2008; $1 \overbrace{}^{2}, 2$ 우 (MZDR), 4 우 (AE), same locality, 23.VI.2008; 1 (AE), same locality, 9.VII.2009, all leg. N.O. Kostyanaya; $1 \mathrm{O}^{7}, 4$ 우 (AE), Neklinovsk Distr., near Merzhanovo, shelter belt, leaf litter, 6.VI.2009, leg. A.P. Evsyukov \& D.D. Khisametdinova; $2 O^{7} \sigma^{7}, 2$ 우 (AE), Rostov-on-Don City, 5.IV.2008; $10^{7}, 5$ 우 (AE), same locality, 18.IX.2008, all leg. A.P. Evsyukov; $10^{3}, 3$ 오 (AE), same locality, 14.IV.2010, leg. E.A. Bortnikov.

OTHER MATERIAL. 1 (AE), Republic of Kalmykia, Gorodovikovsk, 28.III.2012, leg. O.A. Evsyukova; 1 ㅇ (AE), Republic of Adygea, Kamyshanova Polyana, 24.VI.2011, leg. D.D. Khisametdinova.

REMARKS. Above are the northernmost records of this species. Earlier, S. kordylamythrum has only been known from the Caucasus: Krasnodar Province, Stavropol Province, Abkhazia, Georgia and Azerbaijan [Lohmander, 1936, Golovatch \& Matyukhin, 2011]. This species could have arrived to the Rostov-on-Don Region from the northwestern Caucasus via shelter belts and/or through ornithochory, to the Republic of Kalmykia from the neighbouring Daghestan, more likely through ornithochory. In the northern Caucasus, S. kordylamythrum has been recorded in bird nests [Golovatch \& Matyukhin, 2011]. It is noteworthy that the northernmost samples, both from the Rostov-on-Don Region and Kalmykia, tend to be considerably smaller than those from the Caucasus proper (Figs $1 \& 2$ ). Apparently, this reflects more difficult conditions the species is subjected to at the northern periphery of its distribution.

\section{Strongylosoma jaqueti Verhoeff, 1898} Map 1.

MATERIAL. $1 \overbrace{}^{7}, 1$ (ZMUM), 1 (MZDR), 19 (AE), Rostov-on-Don Region, Neklinovsk Distr., near Grecheskie Roty, bank of Mius River, under stones, 2.V.2009, leg. I.L. Gorbenko.

REMARKS. This is the first record of this species in Russia. Previously, it has been reported from Bulgaria, Romania and the Ukraine. At least in the Ukraine, $S$. jaqueti has been considered as an introduced faunal element [Chornyi \& Golovatch, 1993]. Anthropochory seems to be true for the Rostov-on-Don Region as well.

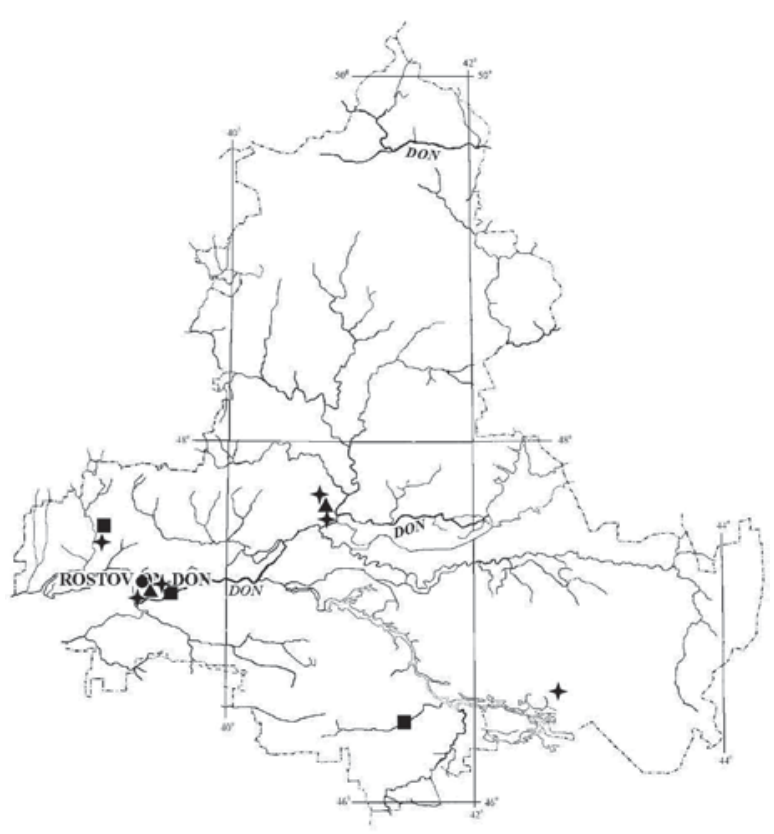

Map 2. Distribution of millipedes in the Rostov-on-Don Region: square - Polydesmus stuxbergi; circle — Brachydesmus jubatus; triangle - Schizoturanius dmitriewi; cross - Nopoiulus kochii.

Карта 2. Распространение двупарноногих многоножек на территории Ростовской области: квадрат — Polydesmus stuxbergi; круг — Brachydesmus jubatus; треугольник Schizoturanius dmitriewi; крест — Nopoiulus kochii.

\section{Oxidus gracilis (C.L. Koch, 1847)}

MATERIAL. 10 우 (MZDR), 2 우, 31 juv. (AE), Rostov-onDon Region, Rostov-on-Don City, Botanical Garden, greenhouse, 19.I.2011, leg. D.D. Khisametdinova \& V.Yu. Shmatko; $1 \sigma^{7}, 3$ 우 (ZMUM), $2 \bigcirc^{\top} \sigma^{\top}, 7$ 우 (AE), same locality, 28.IX.2011, leg. V.Yu. Shmatko \& A.V. Malinovkin.

REMARKS. This subcosmopolitan species of East Asian origin has long become established in man-made habitats, mainly greenhouses, across Europe [Stoev et al., 2010].

\section{Family POLYDESMIDAE}

\section{Polydesmus stuxbergi Attems, 1907}

Figs 3 \& 4; Map 2.

MATERIAL. $2 \sigma^{\top} \sigma^{\top}, 2$ o 2 (ZMUM), $4 \sigma^{\top} \sigma^{\top}, 5$ 오 (AE), Rostov-on-Don Region, Salsk Distr., Salsk, 2.V.2004, leg. N.O. Kostyanaya; 1 ( (AE), Rostov-on-Don City, 22.V.2009; 1 O', 2 우 (AE), same locality, 29.XI.2009; 5 oㅇ (AE), same locality, 26.IV.2011, all leg. D.D. Khisametdinova; 2 우 (MZDR), 2 우 (AE), same locality, 14.IV.2010, leg. E.A. Bortnikov; 1ㅇ (AE), Neklinovsk Distr., near Morskoy Chulek, steppe, under stones, 18.V.2010, leg. A.P. Evsyukov, D.D. Khisametdinova \& V.Yu. Shmatko; $2 \sigma^{\top} \sigma^{T}$ (AE), Neklinovsk Distr., near Troitskoe, bank of Mius River, under bark, 2.V.2011, leg. D.D. Khisametdinova \& Yu.V. Kochetov.

OTHER MATERIAL. $1 \overbrace{}^{7}, 3$ oq (ZMUM), Ukraine, Crimea, Mt Villya-Burun, Villyaburunskaya Cave, traps, 12.V.200812.IX.2010; $5 \sigma^{\top} \sigma^{7}, 5$ 우 (ZMUM), same locality, 19.VII.200417.VII.2006, all leg. A.G. Koval. 

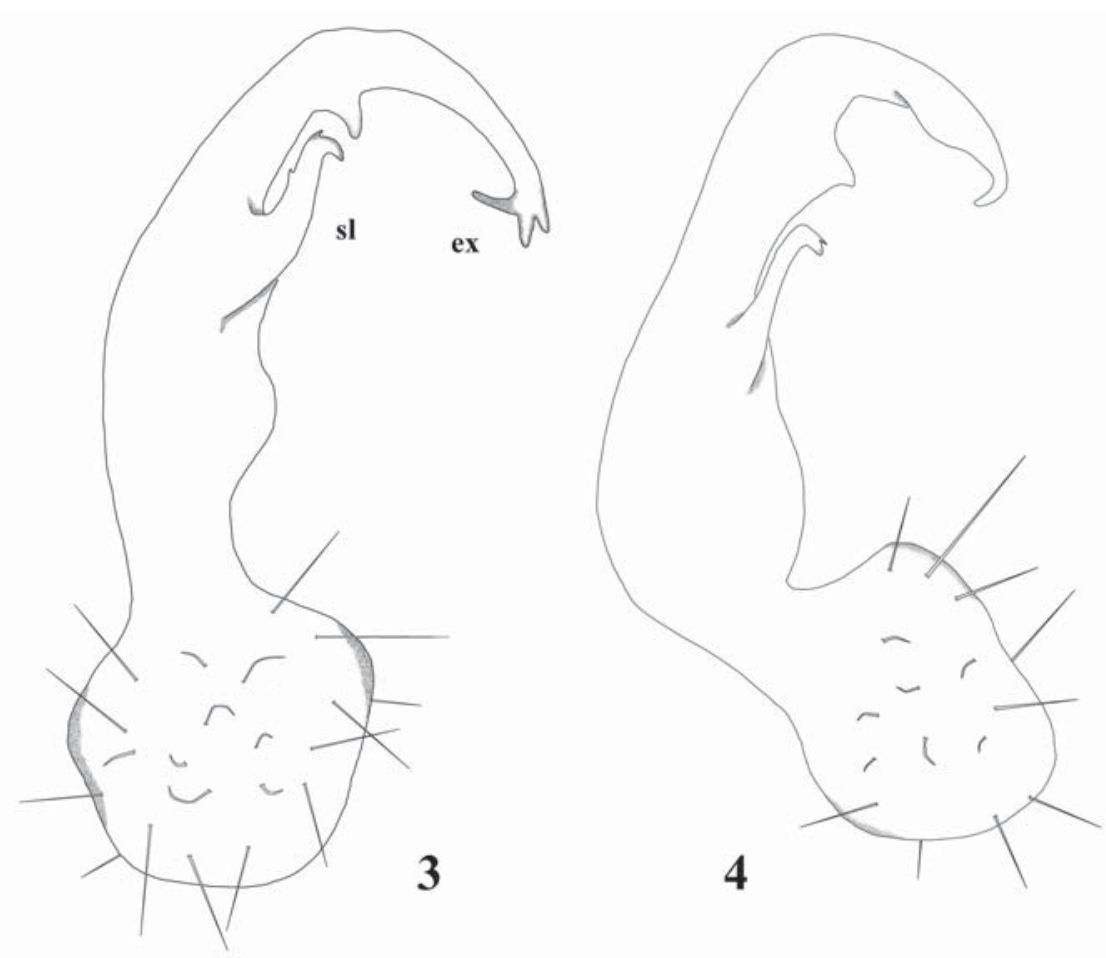

Figs 3 \& 4. Polydesmus stuxbergi, right gonopod from samples from the Rostov-on-Don Region (left) and the Crimea (right), respectively. Del. A. Evsyukov, not to scale.

Рис. 3 и 4. Polydesmus stuxbergi, правый гонопод экземпляров соответственно из Ростовской области (слева) и Крыма (справа). Рисунок А. Евсюкова, без масштаба.

REMARKS. Above are the first records of this species in Russia. Earlier, P. stuxbergi has been recorded in the Crimea and near Kharkov, Ukraine [Golovatch, 1990]. In the Rostov-on-Don Region, P. stuxbergi has only been found in anthropogenous habitats.

The gonopods of $\sigma^{7}$ examples from the Rostov-onDon Region appear to slightly differ compared to material from the Crimea, the terra typica [Attems, 1907]. Thus, the solenomere (sl) of $\sigma^{7} \sigma^{7}$ from the Rostov-onDon Region shows an additional denticle, whereas the exomere (ex) ends up with three teeth as opposed to one tooth in specimens from the Crimea (Figs $3 \& 4$ ).

\section{Brachydesmus jubatus Attems, 1907}

Figs 5 \& 6; Map 2.

MATERIAL. $1 \sigma^{7}, 1$ (ZMUM), $10^{7}, 1$ (AE), Rostov-onDon Region, Myasnikovsk Distr., near Nedvigovka, gully, leaf litter, 14.IV.2002, leg. A.P. Evsyukov \& D.D. Khisametdinova.

REMARKS. This is the first record of this species in Russia (Figs 5 \& 6). It has hitherto been reported from the Crimea, Ukraine, northern Turkey and Dobrogea, Romania [Golovatch, 1984, 1990, 2008].

Schizoturanius dmitriewi (Timotheew, 1897)

$$
\text { Map } 2 .
$$

MATERIAL. 1 \&, 5 juv. (MZDR), 6 juv. (AE), Rostov-on-Don Region, Ust'-Donetsk Distr., near Kanygin, bank of Sukhoi Donets
River, flood-land forest, leaf litter, 15.VI.2009; 1 क (AE), same locality, 16.VI.2009, all leg. A.P. Evsyukov \& D.D. Khisametdinova; 1 o (AE), Myasnikovsk Distr., near Nedvigovka, bank of Mertvyi Donets River, pitfall traps, 5-10.XI.2009, leg. A. Golovanov.

REMARKS. Above are the southernmost records of this species which is endemic to the forested steppe belt of the Eastern European Plain both within Russia and the Ukraine [Golovatch, 1984]. It is most abundant in the steppe east of the Dnepr, in the lower Don River basin [Wytwer et al., 2009].

\section{ORDER JULIDA}

\section{Family BLANIULIDAE}

Choneiulus palmatus (Nĕmec, 1895)

MATERIAL. $2 O^{\top} O^{\top}, 2$ 오 (MZDR), $4 O^{\top} \sigma^{\top}, 24$ 오 (AE), Rostov-on-Don City, Botanical Garden, greenhouse, 19.I.2011, leg. D.D. Khisametdinova \& V.Yu. Shmatko.

REMARKS. In the Region, this anthropochore species of European origin [Golovatch, 1984, 1992; Stoev et al., 2010] inhabits only a greenhouse in the city of Rostov-on-Don, definitely representing an introduction.

Blaniulus guttulatus (Fabricius, 1798)

MATERIAL. 1 ( den, greenhouse, 1.IX.2011, leg. D.D. Khisametdinova; $3 \sigma^{7} \sigma^{7}, 3$ 

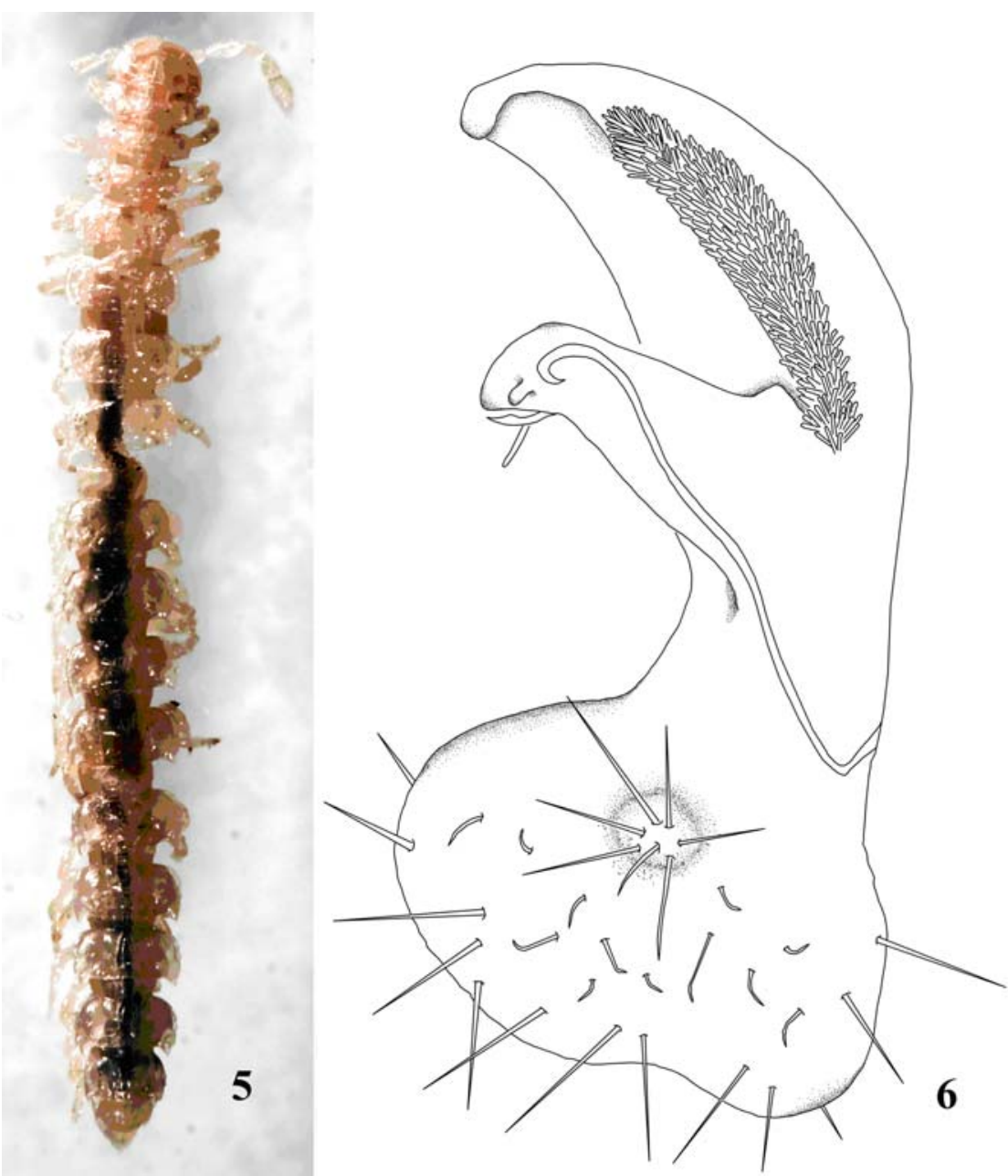

Figs $5 \& 6$. Brachydesmus jubatus, habitus in dorsal view (left) and right gonopod in mesal view (right), respectively. Photo by Alexey Tikhonov, del. A. Evsyukov, both not to scale.

Рис. 5 и 6. Brachydesmus jubatus, соответственно общий вид сверху (слева) и правый гонопод изнутри (справа). Фото Алексея Тихонова и рисунок А. Евсюкова, оба без масштаба.
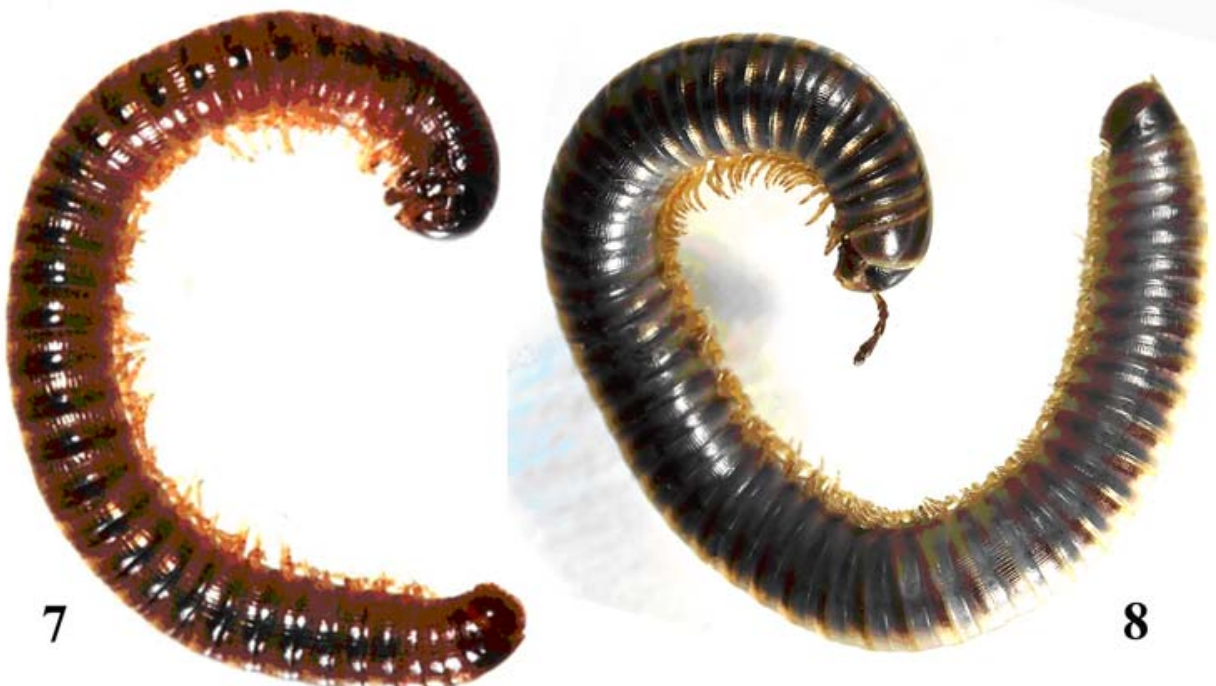

Figs 7 \& 8. Cylindroiulus cambio and Megaphyllum transsylvanicum, habitus (left) and habitus (right) in lateral view, respectively. Photos by Alexey Tikhonov. Photographed not to scale.

Рис. 7 и 8. Cylindroiulus cambio и Megaphyllum transsylvanicum, соответственно общий вид (слева) и общий вид (справа). Фото Алексея Тихонова. Без масштаба. 


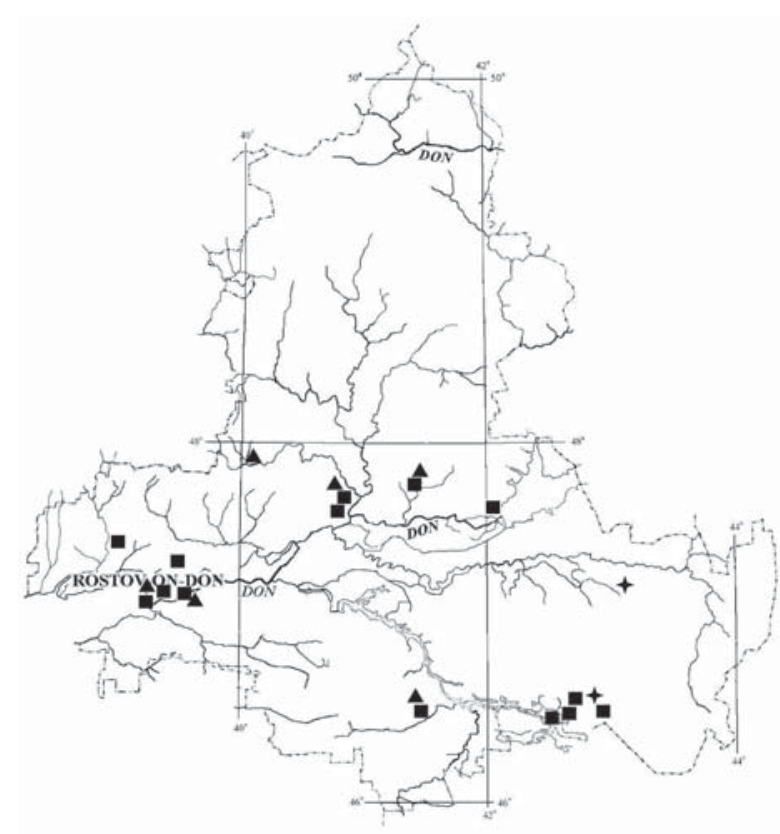

Map 3. Distribution of millipedes in the Rostov-on-Don Region: square - Cylindroiulus cambio; triangle - Brachyiulus jawlowskii; cross - Unciger transsilvanicus.

Карта 3. Распространение двупарноногих многоножек на территории Ростовской области: квадрат - Cylindroiulus cambio; треугольник - Brachyiulus jawlowskii; крест - Unciger transsilvanicus.

우 (MZDR), $9 \odot^{7} \odot^{7}, 3$ 우 (AE), same locality, 28.IX.2011, leg. V.Yu. Shmatko \& A.V. Malinovkin.

REMARKS. In the Region, this species is still another anthropochore of European origin which has only been found in a greenhouse in the city of Rostov-onDon.

$$
\begin{gathered}
\text { Nopoiulus kochii (Gervais, 1847) } \\
\text { Map } 2 .
\end{gathered}
$$

MATERIAL. $1 \sigma^{r}, 1$ (AE), Rostov-on-Don Region, Rostovon-Don City, tree, under bark, 9.V.2002, leg. A.P. Evsyukov; 2 우 (AE), Ust'-Donetsk Distr., near Kanygin, flood-plain forest, 11.X.2008, leg. A.V. Tikhonov; $1 \sigma^{\top}, 1$ O (AE), Neklinovsk Distr. near Merzhanovo, 26.X.2008, leg. A.P. Evsyukov \& D.D. Khisametdinova; 1 (AE), Neklinovsk Distr., near Grecheskie Roty, bank of Mius River, under stones, 2.V.2009, leg. I.L. Gorbenko; $1 \sigma^{7}, 1$ juv. (MZDR), Ust'-Donetsk Distr., near Kanygin, bank of Sukhoi Donets River, flood-land forest, leaf litter, 15.VI.2009, leg. A.P. Evsyukov. \& D.D. Khisametdinova; 1 \%7, 3 우 (AE), Orlovsk Distr., Volochaevskiy, 29.IX.2009, leg. A.V..Tikhonov, V.Yu. Shmatko \& A.V. Malinovkin; $2 \sigma^{7} \sigma^{7}, 2$ 우 (AE), Rostov-on-Don City, 14.IV.2010, leg. E.A. Bortnikov; $6 \sigma^{\top} \sigma^{\top}, 2$ 우 (AE), Orlovsk Distr., near Runnyi, sand, 2.V.2010, leg. A.P. Evsyukov; $2 \bigcirc^{\top} \sigma^{7}, 1$ + (AE), Neklinovsk Distr., near Morskoy Chulek, steppe, under stones, 18.V.2010, leg. A.P. Evsyukov, D.D. Khisametdinova \& V.Yu Shmatko; $1 \sigma^{\top}, 8$ $9+$ (AE), Myasnikovsk Distr., near Nedvigovka, Kamennaya gully, under stones, 17.IV.2011, leg. D.D. Khisametdinova \& E.A. Bortnikov; $6 \sigma^{\top} \sigma^{\gamma}, 16$ 우, 1 juv. (AE), same locality, 21.IV.2011, leg. D.D. Khisametdinova, Yu.V. Kochetov \& E.A. Bortnikov; $2 \sigma^{\top} \sigma^{\top}, 10$ 우 (AE), Ust'-Donetsk Distr., near Krymskiy, bank of a brook, 1.V.1997, leg. Z.G. Prishutova.
REMARKS. This ubiquitous species of Caucasian origin is an anthropochore very common in the Region and beyond [Golovatch \& Enghoff, 1990].

\section{Family JULIDAE}

Cylindroiulus cambio Korsós \& Read, 1994

Fig. 7; Map 3.

MATERIAL. $1 \sigma^{7}, 2$ ㅇ (ZMMU), $4 \sigma^{7} \sigma^{7}, 19$ (AE), Rostov-onDon Region, Myasnikovsk Distr., near Nedvigovka, Kamennaya gully, steppe, under stones, 12.IV.2009, leg. A.P. Evsyukov \& D.D. Khisametdinova; $10^{7}, 3$ 우오 (MZDR), 3 o $^{7} \sigma^{7}, 6$ 우 (AE), same locality, 17.IV.2011, leg. D.D. Khisametdinova \& E.A. Bortnikov.

REMARKS. This species has been described from the Botanical Garden in Rostov-on-Don [Korsós \& Read, 1994]. In the original description, the authors noted that $C$. cambio could have been introduced to the Garden. Our new records (Fig. 7) in native steppe habitats near Rostov-on-Don are rather evidence of this species being a steppe-dweller while the type could have been taken from a steppe plot in the Garden.

\section{Cylindroiulus truncorum (Silvestri, 1896)}

MATERIAL. $5 \sigma^{7} \sigma^{7}, 5$ 우 (ZMMU), $3 \bigcirc^{7} \sigma^{7}, 7$ ( $\sigma^{7} \sigma^{7}, 43$ of, 2 juv. (AE), Rostov-on-Don City, Botanical Garden, greenhouse, 19.I.2011, leg. D.D. Khisametdinova \& V.Yu. Shmatko; $2 O^{7} O^{7}, 4$ 우, 1 juv. (AE), same locality, 1.IX.2011, leg. D.D. Khisametdinova.

REMARKS. In the Region, this anthropochore species of North African origin has only been found in a greenhouse of the Botanical Garden in Rostov-on-Don. It is widespread across Europe in such synanthropic habitats as greenhouses, gardens, parks etc. [Korsós \& Enghoff, 1990; Chornyi \& Golovatch, 1993; Stoev et al., 2010].

\section{Unciger transsilvanicus (Verhoeff, 1899) Map 3.}

MATERIAL. $1 \sigma^{\top}$ (AE), Rostov-on-Don City, tree, under bark, 5.IV.2008, leg. A.P. Evsyukov; $2 \sigma^{7} \sigma^{\top}, 3$ 90 (AE), Neklinovsk Distr., near Morskoy Chulek, steppe, under stones, 18.V.2010, leg. A.P. Evsyukov, D.D. Khisametdinova \& V.Yu. Shmatko; 8 q0 (AE), Neklinovsk Distr., near Troitskoe, bank of Mius River, under bark, 2.V.2011, leg. D.D. Khisametdinova \& Yu.V. Kochetov; 1 $\sigma^{\top}, 1$ (AE), Myasnikovsk Distr., near Nedvigovka, Kamennaya gully, steppe, under stones, 21.IV.2011, leg. D.D. Khisametdinova, Yu.V. Kochetov \& E.A. Bortnikov.

REMARKS. This southeastern European species [Golovatch, 1990] reaches in the east as far as the northwestern Caucasus (Georgievsk).

\section{Brachyiulus jawlowskii Lohmander, 1928 Map 3.}

MATERIAL. $3 \sigma^{7} \sigma^{7}, 7$ 90,1 juv. (AE), Rostov-on-Don Region, Ust'-Donetsk Distr., near Krymskiy, bank of a brook, 01.V.1997; $1 \Im^{7}, 2$ 우, 1 juv. (AE), same locality, forest in a ravine, 02.V.1997; 3 OO, 1 juv. (AE), same locality, artificial coniferous plantation, 02.V.1997, all leg. Z.G. Prishutova; $1 \sigma^{7}$ (AE), Myasnikovsk Distr., near Nedvigovka, shelter belt, pitfall traps, 910.X.2001, leg. A.P. Evsyukov \& D.D. Khisametdinova; $3 \sigma^{7} \sigma^{7}, 2$ 
우 (AE), Orlovsk Distr., near Pravoberezhnyi, under dung, 14.IV.2008, leg. A.P. Evsyukov; 1 q (AE), Rostov-on-Don City, 14.IV.2010, leg. E.A. Bortnikov; $20^{\top} \sigma^{7}, 5$ 우 (MZDR), Orlovsk Distr., Volochaevskiy, 2.V.2010, leg. A.P. Evsyukov \& D.D. Khisametdinova; $2 \sigma^{7} \sigma^{7}, 2$ 우 (AE), Neklinovsk Distr., near Troitskoe, bank of Mius River, under bark, 2.V.2011, leg. D.D. Khisametdinova \& Yu.V. Kochetov.

REMARKS. This species is subendemic to the Eastern European Plain [Golovatch, 1984; Chornyi \& Golovatch, 1993], reaching eastern Poland in the west and being especially abundant in the steppe belt east of the Dnepr, in the lower Don River basin [Wytwer et al., 2009].

\section{Megaphyllum rossicum (Timotheew, 1897) Map 4.}

MATERIAL. 6 우 (AE), Rostov-on-Don Region, Ust'-Donetsk Distr., near Krymskiy, bank of a brook, 01.V.1997; $2 O^{7} O^{7} 13$ 우 (AE), same locality, forest in a ravine, 02.V.1997, all leg. Z.G. Prishutova; $1 \mathrm{O}^{7}, 2$ 우 (AE), delta of Don River, 13.IV.2003, leg. V.A. Minoranskiy; 3 우 (AE), Orlovsk Distr., near Runnyi, steppe, 15.V.2003; $1 \mathrm{O}^{7}, 2$ 아 (AE), same locality, 27.VII.2003; $1 \mathrm{O}^{7}$ (AE), same locality, 07.V.2004, all leg. Z.G. Prishutova; $3 \sigma^{7} \sigma^{7}, 6$ O (AE), Salsk Distr., near Salsk, artificial forest plantation, 3.V.2008, leg. A.P. Evsyukov \& D.D. Khisametdinova; $3 \sigma^{7} \sigma^{7}, 2$ 우 (AE), Orlovsk Distr., Volochaevskiy, 15.IV.2008, leg. A.P. Evsyukov; 87 $\sigma^{7} \sigma^{7}, 156$ 우, 35 juv. (AE), Orlovsk Distr., near Runnyi, shelter belt, pitfall traps, 17-19.IV.2008; $1 \mathrm{O}^{7}$ (AE), Orlovsk Distr., near Runnyi, bank of Lake Lebyazh'e, steppe, under dung, 2.V.2008, all leg. A.P. Evsyukov \& D.D. Khisametdinova; 6 우 (AE), Salsk Distr., Salsk, 16.VI.2008; 2 क⿱, 5 juv. (AE), same locality, 9.VII.2009, all leg. N.O. Kostyanaya; 1 \& (AE), Rodionovo-Nesvetaisk Distr., near Bolshekrepinskaya, leaf litter, 2.VII.2008, leg. D.D. Khisametdinova; 3 우 (AE), Ust'-Donetsk Distr., near Kanygin, flood-plain forest, 11.X.2008, leg. A.V. Tikhonov; $1 \mathrm{O}^{\top}, 2$ 우 (AE), Tsimlyansk Distr., near Tsimlyansk, Donskoi Nature Reserve, forest, sand, 10.X.2008 leg. N.I. Bulisheva; $1 O^{\top}$ (AE), Neklinovsk Distr., near Merzhanovo, 26.X.2008, leg. A.P. Evsyukov \& D.D. Khisametdinova; 14 오 (AE), Myasnikovsk Distr., near Nedvigovka, steppe, under stones, 5.IV.2009, leg. A.P. Evsyukov; 1 O7, 6 우 (AE), same locality, Kamennaya gully, steppe, under stones, 12.IV.2009, leg. A.P. Evsyukov \& D.D. Khisametdinova; 5 O $^{7} \mathrm{O}^{7}, 6$ 우 (AE), Orlovsk Distr., Volochaevskiy, 14.IV.2009, leg. A.P. Evsyukov, D.D. Khisametdinova \& V.Yu. Shmatko; 6 O $^{\top} \mathrm{O}^{2}, 10$ 우, 1 juv. (AE), Orlovsk Distr., near Pravoberezhnyi, bank of Lake Lebyazh'e, steppe, under dung, 15.IV.2009; $2 \sigma^{7} \sigma^{7}, 1$ ㅇ (MZDR), Orlovsk Distr., near Volochaevskiy, steppe, under stones, 15.IV.2009, all leg. A.P. Evsyukov, D.D Khisametdinova \& M.A. Tikhonova; 107 (AE), same locality, 29.IX.2009, leg. A.V. Tikhonov, V.Yu. Shmatko \& A.V. Malinovkin; $1 \mathrm{O}^{\prime}(\mathrm{AE})$, Neklinovsk Distr., near Grecheskie Roty, bank of Mius River, under stones, 2.V.2009, leg. I.L. Gorbenko; 1 ㅇ (AE), Rostov-on-Don City, Botanical Garden, 20.V.2009; 1 (AE), same locality, 24.V.2009, all leg. D.D. Khisametdinova; $1 \mathrm{O}^{7}, 135$ 우 (AE), Ust'-Donetsk Distr., near Kanygin, bank of Sukhoi Donets River, flood-plain forest, leaf litter, 15.VI.2009, leg. A.P. Evsyukov \& D.D. Khisametdinova; 1 ( (AE), Rostov-on-Don City, 14.IV.2010, leg. E.A. Bortnikov; $1 \sigma^{7}$ (AE), Neklinovsk Distr., near Morskoy Chulek, steppe, under stones, 18.V.2010, leg. A.P. Evsyukov, D.D Khisametdinova \& V.Yu. Shmatko; 3 우 (AE), Ust'-Donetsk Distr., near Nizhnekundryuchenskaya, tree, under bark, 23.V.2010, leg. A.V. Tikhonov; 1 ㅇ (AE), same locality, 24.VII.2010, leg. D.D Khisametdinova; $1 \mathrm{O}^{7}, 1$ ( $(\mathrm{AE})$, Orlovsk Distr., near Pravoberezhnyi, Lake Manych-Gudilo, Vodnyi Island, steppe, 27.09.2007; $10^{7}$, 1 + (AE), Ust'-Donetsk Distr., near Krymskiy, artificial coniferous plantation, 02.V.1997, all leg. Z.G. Prishutova; 1 오 (AE), Orlovsk Distr., near Manych, 20.V.2010, leg. E.A. Khachikov.

REMARKS. This species is likewise subendemic to the Eastern European Plain, also common in the northern Caucasus [Golovatch, 1984; Chornyi \& Golo-

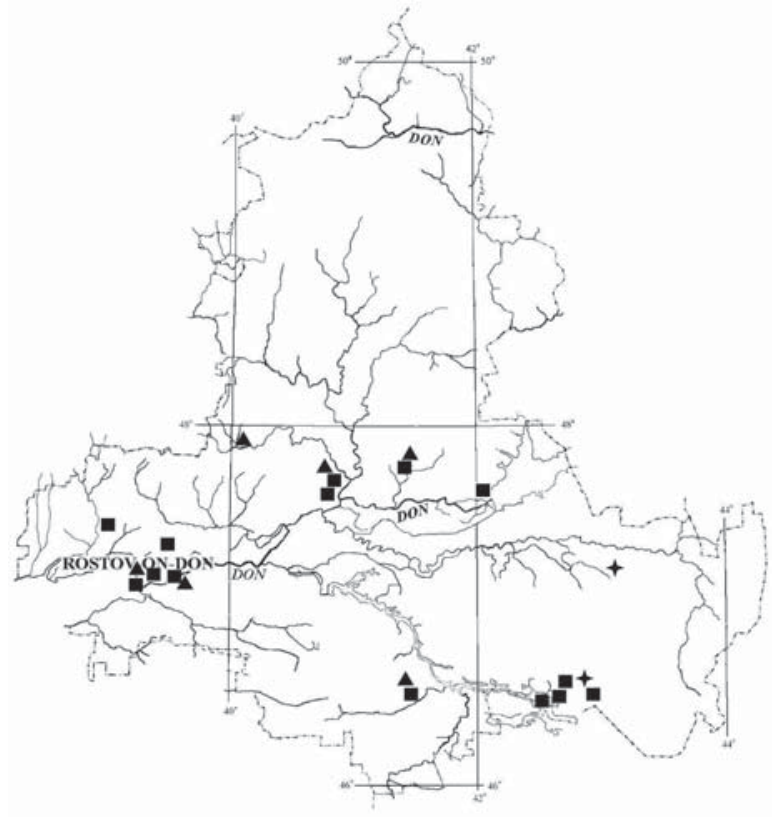

Map 4. Distribution of millipedes in the Rostov-on-Don Region: square - Megaphyllum rossicum; cross - M. transsylvanicum; triangle - Rossiulus kessleri.

Карта 4. Распространение двупарноногих многоножек на территории Ростовской области: квадрат — Megaphyllum rossicum; крест - M. transsylvanicum; треугольник - Rossiulus kessleri.

vatch, 1993]. It is also most abundant in the steppe belt east of the Dnepr, in the lower Don River basin [Wytwer et al., 2009].

\section{Megaphyllum transsylvanicum (Verhoeff, 1897)} Figs 8 \& 9; Map 4.

MATERIAL. $10^{\top}, 3$ ㅇ (AE), Rostov-on-Don Region, Dubovsk Distr., near Prisalskiy, pitfall traps, 21-23.IX.2007, leg. A.V. Adamov \& A.O. Adamova; $10^{7}, 3$ 우 (ZMUM), 5 +o (AE), Orlovsk Distr., near Runnyi, bank of Lebyazh'e Lake, steppe, under dung, 2.V.2008, leg. A.P. Evsyukov \& D.D. Khisametdinova.

REMARKS. Above are the first records of this species in Russia. It is widespread in the Balkans, Romania, Hungary, Moldova and the Ukraine (Odessa, Lvov and Ternopol regions) [Chornyi \& Golovatch, 1993]. The gap between Moldova and the southwestern Ukraine, on the one hand, and the Rostov-on-Don Region, on the other hand, could be accounted for either by undercollecting across the southern Ukraine or through a random introduction to the latter Region. In any event, the Rostov records are currently the easternmost in the distribution of this species. Figs $8 \& 9$ are provided to document the new records, showing the habitus and some details of promere (pr) and opisthomere (op) structure.

\section{Rossiulus kessleri (Lohmander, 1927)} Map 4.

MATERIAL. $1 \sigma^{7}, 10$ 우 (AE), Rostov-on-Don Region, Ust'Donetsk Distr., near Nizhnekundryuchenskaya, 4.VI.2005; 9 우 


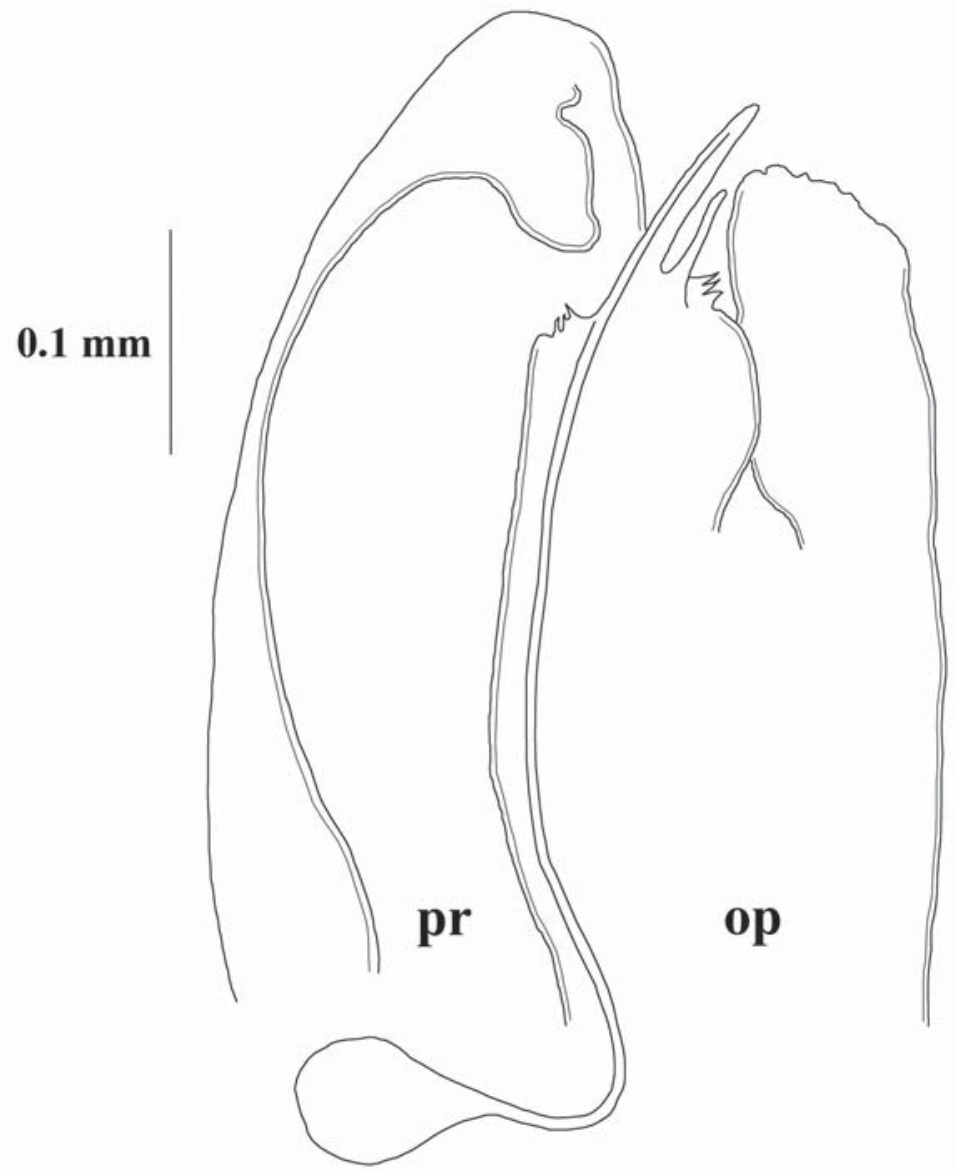

Fig. 9. Megaphyllum transsylvanicum, $0^{7}$ from near Prisalskiy, right gonopods (promere and opisthomere) in submesal view. Del. A. Evsyukov.

Рис. 9. Megaphyllum transsylvanicum, $\mathrm{O}^{7}$ из Присальского, правые гоноподы (промер и опистомер) при виде примерно изнутри. Рисунок А. Евсюкова.

(AE), same locality, 8.VI.2008; $7 \sigma^{7} \sigma^{\top}, 22$ ○ᄋ (MZDR), same locality 14.VI.2008, all leg. O.P. Dobrovol'skiy; $1 \sigma^{7}$ (AE), Krasnyi Sulin Distr., near Krasnyi Sulin, bank of Kundryuchiya River, 5.VI.2007, leg. A.P. Evsyukov; 10 of (AE), Salsk Distr., Salsk, 16.VI.2008, leg. N.O. Kostyanaya; 1 juv. (AE), same locality, artificial forest plantation, 30.IV.2008, leg. A.P. Evsyukov \& D.D. Khisametdinova; 10 우 (AE), Myasnikovsk Distr., near Nedvigovka, Kamennaya gully, 15.V.2008, leg. N.O. Kostyanaya; 1 (AE), Ust'-Donetsk Distr., near Kanygin, bank of Staryi Don River, flood-plain forest, leaf litter, 9.VI.2008, leg. A.V. Tikhonov; 1 (AE), Ust'-Donetsk Distr., near Kanygin, bank of Sukhoi Donets River, flood-plain forest, leaf litter, 16.VI.2009, leg. A.P. Evsyukov \& D.D. Khisametdinova; 9 우 (AE), Myasnikovsk Distr., near Nedvigovka, steppe, under stones, 5.IV.2009, leg. A.P. Evsyukov; 1 (AE), Rostov-on-Don City, 25.V.2009, leg. D.D. Khisametdinova; 2 of (AE), Myasnikovsk Distr., near Nedvigovka, Kamennaya gully, steppe, under stones, 18.IV.2010, leg. E.A. Bortnikov; 1 ( $\mathrm{AE})$, Neklinovsk Distr., near Morskoy Chulek, steppe, under stones, 18.V.2010, leg. A.P. Evsyukov, D.D. Khisametdinova \& V.Yu. Shmatko; 4 우 (AE), Konstantinovsk Distr., near Mikhailovskiy, 23.V.2010, leg. A.V. Tikhonov; 3 90 (AE), Ust'Donetsk Distr., near Nizhnekundryuchenskaya, under bark, 23.V.2010, leg. A.V. Tikhonov; 8 + (ZMMU), bank of Severskiy Donets River, VI.2012, leg. Kakichev.

REMARKS. This species is widespread across the Eastern European Plain, often referred to as its subendemic [Lokshina, 1969; Chornyi, Golovatch, 1993; Golovatch, 1984]. It ranges from near Arkhangelsk in the north to the alpine meadows of North Ossetia, Caucasus in the south, and from slightly east of Minsk, Belarus in the west to the Ural Mountains, Russia in the east. It is also very common in the Rostov-on-Don Region, inhabiting various biotopes, but most abundant in the steppe belt east of the Dnepr, in the lower Don River basin [Wytwer et al., 2009].

\section{Conclusions}

As a result of our studies on the Diplopoda of the Rostov-on-Don Region, the fauna has been revealed to contain at least 17 species. Eleven of them appear to be new to the Region's list, including five which are new to the fauna of Russia: Lophoproctus lucidus (Chalande, 1888), Strongylosoma jaqueti Verhoeff, 1898, Polydesmus stuxbergi Attems, 1907, Brachydesmus jubatus Attems, 1907 and Megaphyllum transsylvanicum (Verhoeff, 1897). Another four species have only been encountered in a greenhouse in the Botanical Garden of Rostov-on-Don. Cylindoiulus cambio Korsós et Read, 1994 may well prove to be endemic to the Region. It is a kind of crossroads where the Caucasian millipede Strongylosoma kordylamythrum Attems, 1898 obviously occurs at the northwestern periphery of its distribution, the Eastern European Schizoturanius dmitriewi (Timotheew, 1897) is at its southern range limit, whereas both Lophoproctus lucidus (Chalande, 1888) and Megaphyllum transsylvanicum (Verhoeff, 1897) 
seem to be at their northeastern range peripheries. Future collecting efforts, as well as faunistic studies on the millipedes of southern Russia and adjacent areas will undoubtedly reveal further interesting novelties.

ACKNOWLEDGEMENTS. We are most grateful to all our colleagues who provided additional material for the present study. We are also deeply obliged to Alexey Tikhonov, Rostov-on-Don, Russia, who skillfully took the pictures.

\section{References}

Attems C. 1907. Myriopoden aus der Krim und dem Kaukasus von Dr. A. Stuxberg gesammelt // Arkiv för Zoologi. Bd.3. No.25. S.1-16.

Chornyi N.G., Golovatch S.I. 1993. [Millipedes (Diplopoda) of the plain territories of the Ukraine]. Kiev. 54 pp. [in Russian, English abstract].

Golovatch S.I. 1984. [The distribution and faunogenesis of millipedes of the USSR European part] // Chernov Yu.I. (ed.) Filogenez i filotsenogenez. Moscow: Nauka Publ. P.92-138 [in Russian]

Golovatch S.I. 1990. On the distribution and faunogenesis of Crimean millipedes (Diplopoda) // Minelli A. (ed.). Proceedings of the 7th International Congress of Myriapodology. Leiden, New York, etc.: E.J. Brill. P.361-366.

Golovatch S.I. 1992. Some patterns in the distribution and origin of the millipede fauna of the Russian Plain // Ber. nat.-med. Vereins Innsbruck. Suppl.10. P.373-383.
Golovatch S.I. 2008. On three remarkable millipedes (Diplopoda) from the Crimea, Ukraine // International Journal of Myriapodology. Vol.1. P.97-110.

Golovatch S.I., Enghoff H. 1990. [The millipede Nopoiulus kochii (Gervais, 1847) in the Caucasus] // Striganova B.R. (ed.). Fauna nazemnykh bespozvonochnykh Kavkaza. Moscow: Nauka Publ. P.114-118 [in Russian].

Golovatch S.I., Matyukhin A.V. 2011. New records of millipedes (Diplopoda), mainly from bird nests, in European Russia // Arthropoda Selecta. Vol.20. No.2. P.115-116.

Gvozdetsky N.A. 1968. [A physiographical regionalization of the USSR]. Moscow: Moscow University Press. 576 pp., 1 map [in Russian].

Korsós Z., Enghoff H. 1990. The Cylindroulus truncorum-group (Diplopoda: Julidae) // Ent. scand. Vol.21. P.345-360.

Lokshina I.E. 1969. [Identification book of the millipedes (Diplopoda) in the plain parts of the USSR European territory]. Moscow: Nauka Publ. 78 pp. [in Russian].

Prishutova Z.G. 1986. [Millipedes of the south of the Rostovon-Don Region] // Sokolov V.E. (ed.). Vsesouyznoe soveshchanie po probleme kadastra i ucheta zhivotnogo mira. Tezisy dokladov. Moscow: Rosagropromizdat. P.489-491 [in Russian].

Stoev P., Zapparoli M., Golovatch S., Enghoff H., Akkari N., Barber A. 2010. Myriapods (Myriapoda). Chapter 7.2. // BioRisk. Vol.4. No.1. P.97-130.

Wytwer J., Golovatch S.I., Penev L. 2009. Variation in millipede (Diplopoda) assemblages in oak woodlands of the Eastern European Plain // Soil Organisms. Vol.81. No.3. P.791-813.

Responsible editor K.G. Mikhailov 